\title{
Nonlinear excitations and phonons in alternating chains with mixed CDW-BOW ground state
}

\author{
S.-L. DRECHSLER ${ }^{*}$, J. MALEK ${ }^{*},(1)$ and M. LAVRENTIEV ${ }^{* *}$
}

*Institut für Festkörper- und Werkstofforschung Dresden e.V., Helmholtzstr. 20, 01069 Dresden, Germany

${ }^{* *}$ Institute of Inorganic Chemistry, Novosibirsk, Russia

\begin{abstract}
Two-component nonlinear excitations as various kinds of polarons and solitons as well as optical phonons have been examined self-consistently within a two-band SSH-Holsteintype model for alternating periodic and open chains of molecular A- and atomic B-sites. Analyzing experimental data of $\mathrm{CuO}_{3}$ and $\mathrm{MX}$-chain compounds within the framework of this model, the importance of internal modes for a complete description of such Peierls systems is emphasized.
\end{abstract}

\section{Introduction.}

Alternating chains with diagonal, off-diagonal, intra- and inter-site electron-phonon interaction and simple rational, but non-half filled bands are characterized by a commensurate mixed CDW-BOW ground state and a rich variety of nonlinear defect states [1-5]. Here, we report on results obtained for the 3/4-filled band case (i.e. 1/2-filled antibonding band), in particular on irrational solitonic charges as well as the nature of the inhomogeneous ground state of differently charged open chains (usually with A-molecules as end groups) investigated self-consistently by the method outlined in Sec. 2 .

2. The model, method, and estimation of parameters.

The electronic structure related to $\mathrm{CuO}_{3}$-chains in $\mathrm{YBa}_{2} \mathrm{Cu}_{3} \mathrm{O}_{7}$ can be modelled by a structure of chains with alternating $\mathrm{O}$-ions and $\mathrm{CuO}_{2}$-molecules and effective interaction parameters. The chain bands are formed of $p \sigma$-states at the $\mathrm{O}$-sites and antibonding molecular $d p \sigma$-states at the $\mathrm{CuO}_{2}$ sites. Their Hamiltonian matrix consists of two on-site energies $\varepsilon_{A}$ and $\varepsilon_{B}$ (of the molecular site and the $\mathrm{O}$-site, respectively) and one transfer integral $t$. Here, two kinds of deformation are considered: symmetric $z$-displacements $u=z_{2}-z_{1}$ of the two molecular O-ions, and longitudinal displacements $y$ of all chain sites $m: v_{m}=y_{m+1}-y_{m}$; (see Fig. 1). Thus, we adopt the effective AB-Hamiltonian

$$
\begin{aligned}
H & =H_{e l}+H_{l a t}=\sum_{n, s} \varepsilon_{n} c_{n, s}^{\dagger} c_{n, s}-t_{n, n+1}\left(c_{n+1, s}^{\dagger} c_{n, s}+\text { h.c. }\right)+ \\
& +\frac{1}{2} \sum_{m \in A}\left(K_{v}\left(v_{m}-v\right)^{2}+K_{u}\left(u_{m}-u\right)^{2}+M_{u} \dot{u}_{m}^{2}+M_{A} \dot{v}_{m}^{2}\right)+\frac{1}{2} \sum_{m \in B}\left(K_{v}\left(v_{m}-v\right)^{2}+M_{B} \dot{v}_{m}^{2}\right)
\end{aligned}
$$

where the off-site and on-site terms read for $A$ and B-sites, respectively

$$
t_{m, m+1}=\left\{\begin{array}{r}
-\left[t_{0}-\gamma_{1} u_{m}-\gamma_{2} v_{m}\right] \\
t_{0}-\gamma_{1} u_{m+1}-\gamma_{2} v_{m}
\end{array} \quad, \quad \varepsilon_{m}=\left\{\begin{array}{l}
\varepsilon_{A}-\alpha_{A} u_{m}-\beta_{A}\left(v_{m}+v_{m-1}\right) \\
\varepsilon_{B}-\alpha_{B}\left(u_{m+1}+u_{m-1}\right)-\beta_{B}\left(v_{m}+v_{m-1}\right) .
\end{array}\right.\right.
$$

(1) 


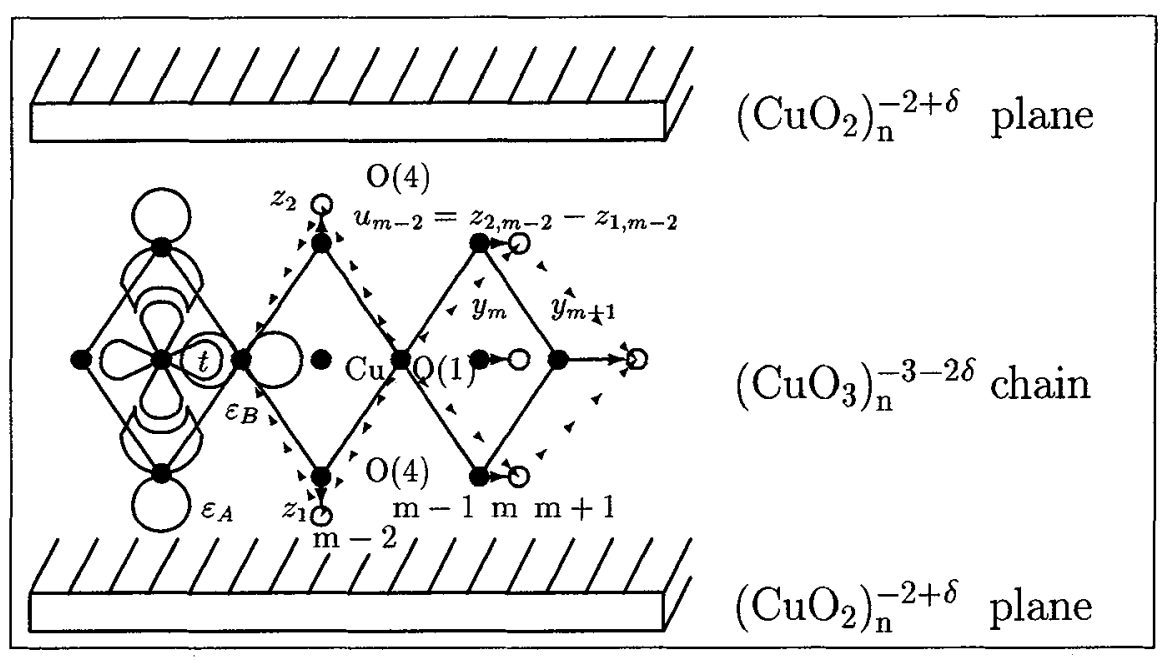

Fig. 1.- Schematical view of a $\mathrm{CuO}_{3}$-chain between two $\mathrm{CuO}_{2}$-planes of the $\mathrm{HTSC} \mathrm{YBa}_{2} \mathrm{Cu}_{3} \mathrm{O}_{7}$. The plane-chain charge transfer is $\delta \approx 1 / 4$. In the case of the $\mathrm{Ca}(\mathrm{Sr})_{2} \mathrm{CuO}_{3}$ compound [6] there are no planes and the apical oxygens $\mathrm{O}(4)$ are embedded in $\mathrm{Ca}(\mathrm{Sr})$-layers and $\delta=0.5$. Possible displacements taken into account within the present model are denoted by arrows.

Experimentally, the band filling ratio $\nu$ of the antibonding chain band $a^{*}$ in $\mathrm{YBa}_{2} \mathrm{Cu}_{3} \mathrm{O}_{7}$ is not precisely known. Therefore, we considered several values, $\nu=1 / 2,1 / 3,1 / 4$ and $1 / 5$ giving equivalent results in the strong coupling case [3]. For $\mathrm{Ca}_{2-x} \mathrm{Sr}_{x} \mathrm{CuO}_{3}$ as for the $\mathrm{MX}$-chains this band is certainly half-filled. The LCAO-deformational study of $\mathrm{YBa}_{2} \mathrm{Cu}_{3} \mathrm{O}_{7}$, presuming an elongation of apical oxygens by $0.1 \AA$ in z-direction, yields $t_{0}=1.67 \mathrm{eV}, \gamma_{1}=1.32 \mathrm{eV} / \AA, \Delta \varepsilon=\varepsilon_{A}-\varepsilon_{B}=1.7 \mathrm{eV}, 2 \alpha_{B}=\alpha_{A}=-0.816$ $\mathrm{eV} / \AA$, and from phonon modes at $505 \mathrm{~cm}^{-1}$ and $400 \mathrm{~cm}^{-1}$ we get $K_{u}=7.78 \mathrm{eV} / \AA^{2}$ and $K_{y}=4.87$ $\mathrm{eV} / \AA^{2}$. For $\beta_{A, B}$ we adopt values of the same order as used in [4] for the related $\mathrm{MX}$-chains. Finally, due to the approximate "symmetry" of a $\mathrm{CuO}_{4}$ plaquette we expect $\gamma_{2} \approx \gamma_{1}$. The Hamiltonian of Eqs. (1-2) is a generalization of the two-band (one-component) Hamiltonian proposed by Bishop et al. for the MX-chains [4] and that of Ref. [1] with respect to diagonal inter-site interaction.

Two self-consistency equations for the displacement patterns are obtained from the variation of the total energy functional with respect to $u_{m}$ and $v_{m}$ :

$$
\begin{aligned}
& u_{m}=u+\frac{1}{K_{u}}\left[\alpha_{A} P_{m, m}+\alpha_{B}\left(P_{m-1, m-1}+P_{m+1, m+1}\right)+2 \gamma_{1}\left(P_{m, m+1}-P_{m-1, m}\right)\right] \\
& v_{m}=v+\frac{1}{K_{v}}\left[\left\{\begin{array}{c}
\beta_{A} \\
\beta_{B}
\end{array}\right\} P_{m, m}+\left\{\begin{array}{c}
\beta_{B} \\
\beta_{A}
\end{array}\right\} P_{m+1, m+1}-2 \gamma_{2}(-1)^{m} P_{m, m+1}\right], \text { for }\left\{\begin{array}{l}
m \epsilon A \\
m \in B
\end{array}\right\}
\end{aligned}
$$

where $P_{m, m^{\prime}}$ denotes the bonding matrix and the core parameters $u$ and $v$ play the role of Lagrangemultipliers for the ground state constraint of constant longitudinal and transverse chain size and they are fixed afterwards evaluating excited states:

$$
\begin{aligned}
& u=\frac{-2}{N K_{u}} \sum_{m \epsilon_{A}}\left[2 \gamma_{1}\left(P_{m, m+1}-P_{m-1, m}\right)+\alpha_{A} P_{m, m}+\alpha_{B}\left(P_{m-1, m-1}+P_{m+1, m+1}\right)\right], \\
& v=\frac{-2}{N K_{v}}\left[\beta_{A} N_{A}+\beta_{B} N_{B}-\gamma_{2} \sum_{m}(-1)^{m} P_{m, m+1}\right],
\end{aligned}
$$

where $N$ stands for the chain site number and $N_{A}\left(N_{B}\right)$ is the total number of electrons on $\mathrm{A}(\mathrm{B})$-sites. 

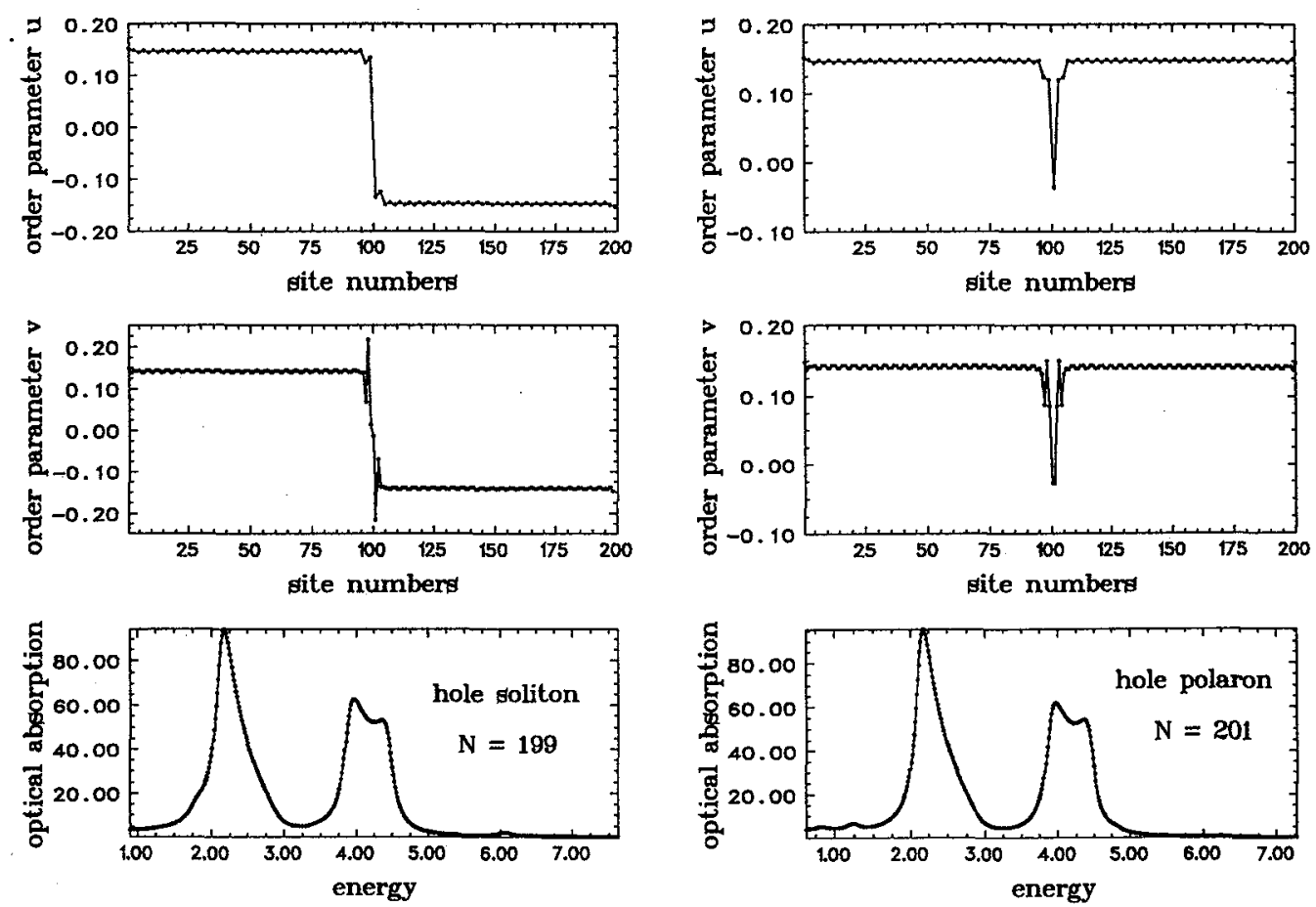

Fig. 2.- Ground state of open alternating chains with odd(even) number of A-sites to the left(right). The staggered order parameters are given in $\AA$, the optical absorption in arbitrary units, and the energy in $\mathrm{eV}$. For the $\mathrm{Ca}_{2-x} \mathrm{Sr}_{x} \mathrm{CuO}_{3}$-parameter set see text.

In other words the mean ground state bond lengths are taken as the reference bond lengths of the expansions in Eq. (2)) Finally, from the variation of the total energy functional with respect to the wave functions one gets the discrete Schrödinger's equation from which the eigenvalues $E_{\mu}$ and eigenstates $\psi_{\mu}(m)$ are obtained for finite $N$-membered rings (or open chains). Eqs. (1-6) can be solved self-consistently by iterations. In the calculations reported below we examined chains with about 200 sites, i.e. the chain length is much larger than the size of defect states (see Fig. 2).

\section{Results.}

\subsection{Phonons.}

For not too strong interactions $\beta_{A}, \beta_{B}$ the Hamiltonian Eqs. (1-2) can be mapped onto the reduced Hamiltonian [1] with two interaction parameters only: $\alpha=\alpha_{A}, \gamma=\gamma_{1}$ provided the same period-4 CDW-BOW state is reproduced. Introducing two corresponding coupling constants $\lambda_{u}=\alpha^{2} / K_{u} t_{0}$ and $\lambda_{v}=\gamma^{2} / K_{v} t_{0}$ the observed optical absorption of $\mathrm{Ca}_{2-x} \mathrm{Sr}_{x} \mathrm{CuO}_{3}$ as well as five optical phonon frequencies calculated within a frozen-phonon type calculation using Eqs. (1-6) can be quantitatively described taking $\lambda_{u}=0.4 \lambda_{v}=0.8, \Delta \varepsilon=1.23 \mathrm{eV}, K_{u}=8.5 \mathrm{eV} / \AA^{2}, K_{v}=18 \mathrm{eV} / \AA^{2}$, and $t_{0}=1.4 \mathrm{eV}$, close to the LCAO-parameter set for $\mathrm{YBa}_{2} \mathrm{Cu}_{3} \mathrm{O}_{7}$ mentioned above. The occurence and polarization of "forbidden" Raman-modes near 500 and $690 \mathrm{~cm}^{-1}$ [6] can be explained quite naturally as a consequence of the period-4 CDW ground state. The accompanying superstructure leads to apical oxygen $\mathrm{O}(4)$ and chain oxygen $\mathrm{O}(1)$ split positions of about $0.15 \AA$, respectively. The same order $(0.13 \AA)$ has been derived from recent EXAFS-data for the apical oxygens in $\mathrm{YBa}_{2} \mathrm{Cu}_{3} \mathrm{O}_{7}$ [7]. Further "forbidden" modes as well as the optical absorption below the Peierls gap of about 1-2eV can be ascribed to defect 
states occuring in open chains kept by A-sites for chemical reasons (see Fig. 2). Since the PtCl-chain compound Raman-data may be fitted succesfully within a pure mechanical model with two quite different ligand spring constants [8], we conclude that the ligands in MX-chains may couple to the $\mathrm{Pt}$-sites like the apical oxygens to the $\mathrm{Cu}$-sites in $\mathrm{CuO}_{3}$-chains. Using Eqs. (1-6) and the experimental in-chain amplitude $v_{0}=0.38 \AA$ we estimate the corresponding weak alternating ligand superstructure having an amplitude of $u_{0}<0.1 \mathrm{eV} / \alpha \sim 0.05 \AA$ which might be overlooked so far.

\subsection{Nonlinear excitations.}

A rich variety of nonlinear defect states as various kinds of polarons and solitons have been found [2,3]. Here, for the limited space, we restrict ourselves to a brief discussion of the soliton charge and the nature of the inhomogeneous ground state of open chains. The ground state of the period-4 CDW-BOW is doubly degenerate with equal charge densities at both B-sites $\rho_{B}$ and two different values $\rho_{A_{t}}$ (maximum) and $\rho_{A_{b}}$ (minimum) at A-sites, respectively. In a reduced notation the effective CDW can be rewritten as $(-f, f,-f, f, \ldots)$, where $f=\rho_{A_{t}}+\rho_{B}$ depends on the model parameters. Summing up the atomic charges over four-site membered blocks we obtained numerically with an accuracy of $10^{-8}$ irrational charges $-f$ and $f$ in units of $|e|$ for the spin-1/2 antisoliton and the soliton, respectively. The charges of spin- 0 objects are simply given by $-f(f) \pm 1$. This result is in accord with the suggestion of Schrieffer [9] that the soliton charge is directly related to the charge per component of the CDW, illustrated in [9] for the 1/3-filled band case and the SSH-model.

Soliton (polaron) centers reside at $\mathrm{B}(\mathrm{A})$-sites. Therefore the ground state of an (open) chain with even (odd) number of $A$-sites has the topological charge \pm 1 or 0 . In the first case there is always a soliton and in the second case, in dependence on the total charge of the chain, a polaron or a quasihomogeneous state result (see Fig. 2). For example, removing an electron from the state depicted in the right sight of Fig. 2, a bipolaron or a soliton-antisoliton pair is produced. In contrast, if one or two electrons are added to the same state mentioned above, a quasi-homogenous period-4-like state arises.

Hole (electron) polarons reside on $A_{b(t)}$-sites. The shape of solitons and antisolitons depends strongly on the occupancy of their intra-gap state. Spin-1/2 solitons (antisolitons) exhibit a smooth spatial dependence of the staggered order parameters.

\section{Acknowledgements.}

We are indebted to the Deutsche Forschungsgemeinschaft for support of the present work under Projects Es 85/1-1 and Dre 269/1-2. Discussions with H. Eschrig and L.S. Cederbaum as well as H. Köppel are gratefully acknowledged.

\section{References}

[1] FISCHER, F., KÖPPEL, H. and CEDERBAUM, L.S., Synthetic Metals 41-43, (1991)3597.

[2] DRECHSLER, S.-L., MALEK, J., KÖPPEL, H., et al. Synthetic Metals 55-57, (1993)4272.

[3] DRECHSLER, S.-L., MALEK, J., and ESCHRIG, H., Synthetic Metals 55-57, 4626(1993).

[4] BISHOP, A.R., GAMMEL, J.T, and PHILLPOT, S.R., Synthetic Metals 29, (1989)F151.

[5] KIM-CHAU UNG, MAZUMDAR, S., and CAMPBELL, D.K., Solid State Commun. 85, (1993) 917.

[6] YOSHDA, M., TAJIMA, S., KOSHIZUKA, N., et al., Phys. Rev. B 44, (1991)11997.

[7] MUSTRE de LEON, J., CONRADSON, S.D., BATISTIĆ, I., et al., Phys. Rev. B 44, (1991)2422.

[8] DEGIORGI, L., WACHTER, P., HARUKI, M., and KURITA, S., Phys. Rev. B 40, (1989)3285.

[9] SCHRIEFFER, J.R., Mol. Cryst. Liq. Cryst. 118, (1985)57. 